\title{
Solution of the Bloch Equations including Relaxation
}

\author{
Eric R. Johnston (D) \\ Department of Chemistry and Biochemistry, The University of North Carolina at Greensboro, Greensboro, NC, USA 27412 \\ Correspondence should be addressed to Eric R. Johnston; erj3681@hotmail.com
}

Received 24 June 2020; Revised 24 July 2020; Accepted 16 August 2020; Published 2 September 2020

Academic Editor: Sharon Ruthstein

Copyright (C) 2020 Eric R. Johnston. This is an open access article distributed under the Creative Commons Attribution License, which permits unrestricted use, distribution, and reproduction in any medium, provided the original work is properly cited.

The magnetization differential equations of Bloch are integrated using a matrix diagonalization method. The solution describes several limiting cases and leads to compact expressions of wide validity for a spin ensemble initially at equilibrium.

\section{Introduction}

In 1949 Torrey used Laplace transforms to provide [1] the first solution of the differential equations proposed by Bloch [2] for the magnetization components of a spin ensemble. His results are somewhat cumbersome and contain some errors. Although the problem is fundamental, a general solution including relaxation does not appear in any of the standard NMR texts, with one partial exception [3]. The problem has been revisited several times employing third-order differential equations $[4,5]$ and Laplace transforms [6] to give unwieldy solutions using somewhat opaque derivations. The first-order differential equations are directly integrated here using a matrix diagonalization method.

\section{Bloch Equations and Their Integration}

The Bloch equations for a collection of identical spins - (1/2) in the frame rotating at $\omega_{\mathrm{rf}}$ are

$$
\frac{\mathrm{d}}{\mathrm{d} t}\left(\begin{array}{c}
M_{x} \\
M_{y} \\
M_{z}
\end{array}\right)=-\mathbf{K}\left(\begin{array}{c}
M_{x} \\
M_{y} \\
M_{z}
\end{array}\right)+\left(\begin{array}{c}
0 \\
0 \\
R_{1} M_{0}
\end{array}\right),
$$

$$
\mathbf{K}=\left(\begin{array}{ccc}
R_{2} & \Delta & 0 \\
-\Delta & R_{2} & \omega_{1} \\
0 & -\omega_{1} & R_{1}
\end{array}\right)
$$

$R_{1}$ and $R_{2}$ are the longitudinal and transverse relaxation rates in $s^{-1}$, and $\Delta=\left|\omega_{0}-\omega_{\mathrm{rf}}\right|$ and $\omega_{1}=\gamma B_{1}$ are the resonance frequency offset and the rf amplitude for a $B_{1}$ field along the $x$-axis, in radians/s. $\gamma$ is the (positive) gyromagnetic ratio, and $M_{0}$ is the equilibrium magnetization.

Defining a magnetization vector $V=\left(\begin{array}{lll}M_{x} & M_{y} & M_{z}\end{array}\right)^{T}$, the integrated solution of equation (1) is

$$
\begin{aligned}
V(t)= & \exp (-\mathbf{K} t)[V(0)-V(s s)]+V(s s)=\mathbf{X} \\
& \cdot \exp (-\mathbf{D} t) \mathbf{X}^{-1}[V(0)-V(s s)]+V(s s),
\end{aligned}
$$

where $\mathbf{K}=\mathbf{X} \mathbf{D X} \mathbf{X}^{-1}$. The problem is, thus, deducing the roots of $\mathbf{K}(=\mathbf{D})$, the matrix $\mathbf{X}$ and inverse $\mathbf{X}^{-1}$ which diagonalize $\mathbf{K}$ and the steady-state magnetization vector $V(s s)$.

2.1. Evaluation of Roots. $|\mathbf{K}-\lambda \mathbf{1}|=0$ gives the following cubic equation:

$$
\begin{gathered}
\lambda^{3}-\left(R_{1}+2 R_{2}\right) \lambda^{2}+\left(R_{2}^{2}+2 R_{1} R_{2}+\Delta^{2}+\omega_{1}^{2}\right) \lambda \\
-\left(R_{1} R_{2}^{2}+R_{1} \Delta^{2}+R_{2} \omega_{1}^{2}\right)=0 .
\end{gathered}
$$

where 
Choosing roots of the form $\lambda_{1}=a, \lambda_{2,3}=b \pm i \Omega$ gives the corresponding cubic equation:

$$
\begin{gathered}
(\lambda-a)\left(\lambda^{2}-2 b \lambda+b^{2}+\Omega^{2}\right)=\lambda^{3}-(a+2 b) \lambda^{2} \\
+\left(b^{2}+2 a b+\Omega^{2}\right) \lambda-a\left(b^{2}+\Omega^{2}\right)=0 .
\end{gathered}
$$

Equating the last term in equations (4) and (5), and with $R_{2}<\omega_{1}$ and/or $\Delta$ in (4) and $b<\Omega$ in (5),

$$
a=R_{1} \cos ^{2} \theta+R_{2} \sin ^{2} \theta \equiv R_{\theta},
$$

using $\tan \theta=\left(\omega_{1} / \Delta\right)$ and $\Omega \equiv\left(\Delta^{2}+\omega_{1}^{2}\right)^{1 / 2}$.

$b$ is obtained by equating the coefficients of the second term in equations (4) and (5):

$$
b=\frac{1}{2}\left(R_{1}+2 R_{2}-R_{\theta}\right)=R_{2}-\frac{1}{2}\left(R_{2}-R_{1}\right) \sin ^{2} \theta .
$$

These expressions agree with Torrey ([1], equation 59) and with Abragam ([3]. p. 70).

For $R_{2}=1 s^{-1}$ and $\omega_{1}$ and/or $\Delta=2 \pi \times 10 \mathrm{~Hz}$, for example, the above approach is valid and avoids the explicit solution of the cubic equation (4).

2.2. Calculation of $\mathbf{X} . \mathbf{X}$ is obtained by evaluating the three cofactors of $\mathbf{K}-\lambda \mathbf{1}$ for $\lambda_{1}=R_{\theta}$ and $\lambda_{2,3}=b \pm i \Omega$. Choosing the third row of $\mathbf{K}-\lambda \mathbf{1}$, the cofactors are

$$
\begin{aligned}
& \text { cofactor } 1=\Delta \omega_{1} \\
& \text { cofactor } 2=-\omega_{1}\left(R_{2}-\lambda\right) \\
& \text { cofactor } 3=\left(R_{2}-\lambda\right)^{2}+\Delta^{2}
\end{aligned}
$$

Omitting all (small) relaxation rate difference terms (which are exactly zero for $R_{1}=R_{2}$ ) and dividing all elements by $\omega_{1}$ gives $\mathbf{X}$ :

$$
\mathbf{X}=\left(\begin{array}{ccc}
\Delta & \Delta & \Delta \\
0 & i \Omega & -i \Omega \\
\frac{\Delta^{2}}{\omega_{1}} & -\omega_{1} & -\omega_{1}
\end{array}\right) .
$$

2.3. Calculation of $\mathbf{X}^{-1} \cdot \mathbf{X}^{-1}$ is formed by constructing the matrix of all cofactors of $\mathbf{X}$, taking the transpose, and dividing by the determinant $|\mathbf{X}|[7]$. The result is

$$
\mathbf{X}^{-1}=\frac{1}{2 \Omega^{2}}\left(\begin{array}{ccc}
\frac{2 \omega_{1}^{2}}{\Delta} & 0 & 2 \omega_{1} \\
\Delta & -i \Omega & -\omega_{1} \\
\Delta & i \Omega & -\omega_{1}
\end{array}\right) \text {. }
$$

These may be rewritten in a more compact form using $\tan \theta=\left(\omega_{1} / \Delta\right)$ and $\Omega$ :

$$
\begin{aligned}
\mathbf{X}=\left(\begin{array}{ccc}
\cos \theta & \cos \theta & \cos \theta \\
0 & i & -i \\
\frac{\cos \theta}{\tan \theta} & -\sin \theta & -\sin \theta
\end{array}\right), \\
\mathbf{X}^{-1}=\frac{1}{2}\left(\begin{array}{ccc}
2 \sin \theta \tan \theta & 0 & 2 \sin \theta \\
\cos \theta & -i & -\sin \theta \\
\cos \theta & i & -\sin \theta
\end{array}\right) .
\end{aligned}
$$

Finally, we calculate the matrix $\mathbf{A}=\mathbf{X} \exp (-\mathbf{D} t) \mathbf{X}^{-1}$ :

$$
\begin{aligned}
& \mathbf{A}=\mathbf{X}\left(\begin{array}{lll}
\exp -R_{\theta} t & & \\
& \exp -b t(c-i s) & \\
& & \exp -b t(c+i s)
\end{array}\right) \\
& \mathbf{X}^{-1} \text {, where } \begin{array}{r}
c=\cos \Omega t \\
s=\sin \Omega t
\end{array} \text {. }
\end{aligned}
$$

The elements of $\mathbf{A}$ are

$$
\begin{aligned}
& A_{11}=\sin ^{2} \theta \exp -R_{\theta} t+\cos ^{2} \theta \exp -b t \cos \Omega t, \\
& A_{22}=\exp -b t \cos \Omega t, \\
& A_{12}=-A_{21}=-\cos \theta \exp -b t \sin \Omega t, \\
& A_{23}=-A_{32}=-\sin \theta \exp -b t \sin \Omega t, \\
& A_{13}=A_{31}=\sin \theta \cos \theta\left(\exp -R_{\theta} t-\exp -b t \cos \Omega t\right), \\
& A_{33}=\cos ^{2} \theta \exp -R_{\theta} t+\sin ^{2} \theta \exp -b t \cos \Omega t .
\end{aligned}
$$

2.4. Steady States. The steady-state magnetizations are found by setting equation (1) to zero and using Cramer's rule [7]:

$$
\begin{aligned}
& M_{x}(\mathrm{ss})=\frac{R_{1} \Delta \omega_{1} M_{0}}{d}, \\
& M_{y}(\mathrm{ss})=\frac{-R_{1} R_{2} \omega_{1} M_{0}}{d}, \\
& M_{z}(\mathrm{ss})=\frac{R_{1}\left(R_{2}^{2}+\Delta^{2}\right) M_{0}}{d},
\end{aligned}
$$

where $d=R_{1} R_{2}^{2}+R_{1} \Delta^{2}+R_{2} \omega_{1}^{2}$.

For $R_{2}<\omega_{1}$ and/or $\Delta$, these may be simplified using equation (6):

$$
\begin{aligned}
& M_{x}(\mathrm{ss}) \longrightarrow\left(\frac{R_{1}}{R_{\theta}}\right) \sin \theta \cos \theta M_{0}, \\
& M_{y}(\mathrm{ss}) \longrightarrow-\frac{R_{1} R_{2} \sin \theta}{R_{\theta} \Omega} M_{0} \approx 0, \\
& M_{z}(\mathrm{ss}) \longrightarrow\left(\frac{R_{1}}{R_{\theta}}\right) \cos ^{2} \theta M_{0} .
\end{aligned}
$$


The integrated solution equation (3) for the initial condition $M_{z}(0)=M_{0}$ is

$$
\begin{aligned}
M_{x}(t)= & A_{13} M_{0}-A_{11} M_{x}(\mathrm{ss})-A_{12} M_{y}(\mathrm{ss}) \\
& -A_{13} M_{z}(\mathrm{ss})+M_{x}(\mathrm{ss}), \\
M_{y}(t)= & A_{23} M_{0}-A_{21} M_{x}(\mathrm{ss})-A_{22} M_{y}(\mathrm{ss}) \\
& -A_{23} M_{z}(\mathrm{ss})+M_{y}(\mathrm{ss}), \\
M_{z}(t)= & A_{33} M_{0}-A_{31} M_{x}(\mathrm{ss})-A_{32} M_{y}(\mathrm{ss}) \\
& -A_{33} M_{z}(\mathrm{ss})+M_{z}(\mathrm{ss}) .
\end{aligned}
$$

In the absence of relaxation, these are the well-known Bloch equations $[8,9]$ :

$$
\begin{aligned}
& M_{x}(t)=A_{13} M_{0}=\sin \theta \cos \theta M_{0}(1-\cos \Omega t), \\
& M_{y}(t)=A_{23} M_{0}=-\sin \theta M_{0} \sin \Omega t, \\
& M_{z}(t)=A_{33} M_{0}=\cos ^{2} \theta M_{0}+\sin ^{2} \theta M_{0} \cos \Omega t .
\end{aligned}
$$

\section{Results}

Limiting forms of equation (3) are discussed in this section.

3.1. Case 1: Resonant Nutation $\Delta=\cos \theta=0, b=\left(R_{1}+\right.$ $\left.R_{2}\right) / 2$, and $M_{z}(0)=M_{0}$.

$$
\begin{gathered}
A_{11}=\exp -R_{2} t, \\
A_{12}=A_{21}=A_{13}=A_{31}=0, \\
A_{22}=A_{33}=\exp -b t \cos \omega_{1} t, \\
A_{23}=-A_{32}=-\exp -b t \sin \omega_{1} t, \\
M_{x}(\mathrm{ss})=M_{x}(t)=0, \\
M_{y}(\mathrm{ss})=-\left(\frac{R_{1}}{\omega_{1}}\right) M_{0}, \\
M_{z}(\mathrm{ss})=\left(\frac{R_{1} R_{2}}{\omega_{1}^{2}}\right) M_{0}, \\
M_{y}(t)=A_{23} M_{0}+M_{y}(\mathrm{ss})\left[1-A_{22}\right]-A_{23} M_{z}(\mathrm{ss}), \\
M_{z}(t)=A_{33} M_{0}+M_{z}(\mathrm{ss})\left[1-A_{33}\right]-A_{32} M_{y}(\mathrm{ss}) .
\end{gathered}
$$

$M_{y}$ and $M_{z}$ interconvert at rate $\omega_{1}$ and decay to (small) steady states.
3.2. Case 2: Free Precession $/ M_{z}$ Relaxation $\omega_{1}=\sin \theta=0$ and $M_{x}(0)=M_{z}(\mathrm{ss})=M_{0}$.

$$
\begin{aligned}
& A_{11}=A_{22}=\exp -R_{2} t \cos \Delta t, \\
& A_{12}=-A_{21}=-\exp -R_{2} t \sin \Delta t, \\
& A_{13}=A_{31}=A_{23}=A_{32}=0, \\
& A_{33}=\exp -R_{1} t,
\end{aligned}
$$

$$
\begin{aligned}
& M_{x}(t)=A_{11} M_{0}=M_{0} \exp -R_{2} t \cos \Delta t, \\
& M_{y}(t)=A_{21} M_{0}=M_{0} \exp -R_{2} t \sin \Delta t, \\
& M_{z}(t)=-A_{33} M_{0}+M_{z}(\mathrm{ss})=M_{0}\left(1-\exp -R_{1} t\right) .
\end{aligned}
$$

$M_{x}$ and $M_{y}$ interconvert at rate $\Delta$ and decay to zero as $M_{z}$ returns to equilibrium.

3.3. Case 3: Spin-Locked Relaxation $M_{x}(0)=\sin \theta M_{0}$, $M_{z}(0)=\cos \theta M_{0}$, and $M_{y}(\mathrm{ss}) \approx 0$.

$$
\begin{aligned}
M_{x}(t)= & A_{11} \sin \theta M_{0}+A_{13} \cos \theta M_{0}-A_{11} M_{x}(\mathrm{ss}) \\
& -A_{13} M_{z}(\mathrm{ss})+M_{x}(\mathrm{ss}), \\
M_{y}(t)= & A_{21} \sin \theta M_{0}+A_{23} \cos \theta M_{0}-A_{21} M_{x}(\mathrm{ss}) \\
& -A_{23} M_{z}(\mathrm{ss}), \\
M_{z}(t)= & A_{31} \sin \theta M_{0}+A_{33} \cos \theta M_{0}-A_{31} M_{x}(\mathrm{ss}) \\
& -A_{33} M_{z}(\mathrm{ss})+M_{z}(\mathrm{ss}) .
\end{aligned}
$$
become

Employing the steady states of equations (17)-(19), these

$$
\begin{aligned}
M_{x}(t)= & \sin \theta M_{0} \exp -R_{\theta} t \\
& +\left(\frac{R_{1}}{R_{\theta}}\right) \sin \theta \cos \theta M_{0}\left[1-\exp -R_{\theta} t\right] \\
M_{y}(t)= & 0 \\
M_{z}(t)= & \cos \theta M_{0} \exp -R_{\theta} t \\
& +\left(\frac{R_{1}}{R_{\theta}}\right) \cos ^{2} \theta M_{0}\left[1-\exp -R_{\theta} t\right]
\end{aligned}
$$

The magnetization vector relaxes to a steady state along the effective field $B_{\text {eff }}=(\Omega / \gamma)[10,11]$.

3.4. Case 4: General Solution $M_{z}(0)=M_{0}$.

Equations (20)-(22) may be recast using the steady states of equation (17)-(19) as

$$
\begin{aligned}
& M_{x}(t)=A_{13} M_{0}+M_{x}(\mathrm{ss})\left[1-\exp -R_{\theta} t\right], \\
& M_{y}(t)=A_{23} M_{0}, \\
& M_{z}(t)=A_{33} M_{0}+M_{z}(\mathrm{ss})\left[1-\exp -R_{\theta} t\right] .
\end{aligned}
$$


These expressions agree with equations (60)-(62) of Torrey [1] and with Abragam [3].

For a weak rf field $\omega_{1}<\Delta\left(\cos \theta \longrightarrow 1, \sin ^{2} \theta \longrightarrow 0\right)$, they reduce to

$$
\begin{aligned}
& M_{x}(t)=\left(\frac{\omega_{1}}{\Delta}\right) M_{0}\left(1-\exp -R_{2} t \cos \Delta t\right), \\
& M_{y}(t)=-\left(\frac{\omega_{1}}{\Delta}\right) M_{0} \exp -R_{2} t \sin \Delta t, \\
& M_{z}(t) \approx M_{0},
\end{aligned}
$$

in agreement with Slichter ([12], p. 35).

3.5. Case 5: Equal Relaxation Rates $R_{\theta}=b=R, M_{z}(0)=M_{0}$. An exact solution of the Bloch equations is given by equations (20)-(22) using the full steady-state expressions (14)-(16). Using the steady states of equation (17)-(19), they become

$$
\begin{aligned}
& M_{x}(t)=\sin \theta \cos \theta M_{0}(1-\exp -R t \cos \Omega t), \\
& M_{y}(t)=-\sin \theta M_{0} \exp -R t \sin \Omega t \\
& M_{z}(t)=\cos ^{2} \theta M_{0}+\sin ^{2} \theta M_{0} \exp -R t \cos \Omega t .
\end{aligned}
$$

\section{Discussion}

Equation (3) describes a number of experimental situations.

4.1. Case 1: Resonant Nutation. Resonant nutation (equations (28) and (29) ) was described by Torrey in his original paper [1]. The effective relaxation rate is the average of the longitudinal and transverse relaxation rates [3].

4.2. Case 2: Free Precession and Relaxation. In the absence of an rf field, the transverse components interconvert and relax to zero (the free induction decay) as the longitudinal component, initially zero, relaxes independently to equilibrium (equation (31)).

4.3. Case 3: Spin-Locked Relaxation. Orientation of the magnetization vector parallel to the effective field suppresses precession and results in a single-exponential approach to equilibrium, affording the longitudinal and transverse relaxation rates using equations (6) and (33) [10, 11].

4.4. Case 4: General Solution. Equation (34) presents in compact form the solutions originally given by Torrey [1] and by Morris and Chilvers [6] as Laplace expressions and the tabulations of Madhu and Kumar [4, 5] for a spin ensemble initially at equilibrium. They are valid provided

$$
R_{2}\left(s^{-1}\right)<\Omega\left(\operatorname{rad} s^{-1}\right)
$$

which holds for most cases of practical interest. In the example given by Madhu and Kumar [4, 5],

$$
\begin{aligned}
& R_{1}=10 s^{-1} \\
& R_{2}=100 s^{-1} \\
& \Delta=\omega_{1}=2 \pi \times 1 \mathrm{kHz} .
\end{aligned}
$$

Accordingly, from equations (6), (7), and (17)-(19),

$$
\begin{aligned}
R_{\theta} & =55 s^{-1}, \\
b & =77.5 s^{-1}, \\
\Omega & =2 \pi \times 1414 \mathrm{~Hz},
\end{aligned}
$$

$$
\begin{aligned}
& M_{x}(s s)=M_{z}(s s)=\frac{M_{0}}{11}, \\
& M_{y}(s s)=-\frac{M_{0}}{(110 \times 2 \pi)} \approx 0 .
\end{aligned}
$$

These values do not appear to reproduce the figures presented in [5].

4.5. Case 5: Equal Relaxation Rates. The solutions (20)-(22) for equal relaxation rates are exact provided the full steady states of equation (14)-(16) are used. The inequality of Case 4 leads to the simpler expressions (36). We note also that setting $\omega_{1}=\sqrt{2} \Delta$ results in $R_{\theta}=b=\left(R_{1}+2 R_{2}\right) / 3$.

4.6. Neglect of Relaxation. For rf amplitude and precession terms which are large compared to relaxation rates equations (23)-(25) pertain. They are useful, for example, in describing selective (on-resonance) excitation with (off-resonance) signal suppression [9] as in the following example (using $\mathrm{Hz}$ units).

\subsubsection{On-Resonance Rotation}

$$
\begin{gathered}
\cos \theta \longrightarrow 0, \\
v_{1} t_{\mathrm{p}}=\frac{1}{4}, \\
M_{y}\left(t_{\mathrm{p}}\right)=-M_{0}, \\
M_{x}\left(t_{\mathrm{p}}\right)=M_{z}\left(t_{\mathrm{p}}\right)=0 .
\end{gathered}
$$

\subsubsection{Off-Resonance Rotation}

$$
\begin{aligned}
\Omega t_{P} & =1, \\
M_{z}\left(t_{P}\right) & =M_{0}, \\
M_{x}\left(t_{P}\right) & =M_{y}\left(t_{P}\right)=0, \\
\Omega t_{p} & =1 \text { requires } \Delta t_{\mathrm{p}}=\frac{\sqrt{15}}{4} \text { so } t_{\mathrm{p}}=\frac{\sqrt{15}}{4 \Delta} \mathrm{sec} .
\end{aligned}
$$


Then, $v_{1}=\left(1 / 4 t_{\mathrm{p}}\right)=(\Delta / \sqrt{15}) \mathrm{Hz}$.

For a $5 \mathrm{KHz}$ offset $t_{\mathrm{p}}=193.6 \mu \mathrm{sec}$ and $v_{1}=1291 \mathrm{~Hz}$.

The on-resonance magnetization is rotated to the $-y$ axis by the rf pulse, whereas the off-resonance magnetization undergoes an excursion that returns it to the $z$-axis.

\section{Conclusion}

The differential equations (1) of Bloch [2] are integrated with a matrix diagonalization method to give the solution equation (3). It correctly describes a number of experimental situations including resonant nutation, free precession and relaxation, and spin-locked relaxation. Equation (3) is exact for the case of equal longitudinal and transverse relaxation rates and leads to the general equation (34) for a spin ensemble initially at equilibrium.

\section{Data Availability}

No data were used to support this study.

\section{Conflicts of Interest}

The author declares that there are no conflicts of interest.

\section{References}

[1] H. C. Torrey, "Transient nutations in nuclear magnetic resonance," Physical Review, vol. 76, no. 8, pp. 1059-1068, 1949.

[2] F. Bloch, "Nuclear induction," Physical Review, vol. 70, no. 78, pp. 460-474, 1946.

[3] A. Abragam, The Principles of Nuclear Magnetism, Clarendon Press, Oxford, UK, 1961.

[4] P. K. Madhu and A. Kumar, "Direct Cartesian-space solutions of generalized Bloch equations in the rotating frame," Journal of Magnetic Resonance, Series A, vol. 114, no. 2, pp. 201-211, 1995.

[5] P. K. Madhu and A. Kumar, "Bloch equations revisited: new analytical solutions for the generalized Bloch equations," Concepts in Magnetic Resonance, vol. 9, no. 1, pp. 1-12, 1997.

[6] G. A. Morris and P. B. Chilvers, "General analytical solutions of the Bloch equations," Journal of Magnetic Resonance, Series A, vol. 107, no. 2, pp. 236-238, 1994.

[7] C. L. Perrin, Mathematics for Chemists, John Wiley and Sons, Hoboken, NJ, USA, 1970.

[8] J. Cavanagh, W. J. Fairbrother, A. G. Palmer, M. Rance, and N. J. Skelton, Protein NMR Spectroscopy, Elsevier Academic Press, London, UK, 2nd edition, 2007.

[9] R. R. Ernst, G. Bodenhausen, and A. Wokaun, Principles of Nuclear Magnetic Resonance in One and Two Dimensions, Oxford University Press, Oxford, UK, 1987.

[10] E. R. Johnston, "Magnetization transfer experiments employing continuous RF fields," Concepts in Magnetic Resonance, vol. 11, no. 2, pp. 89-95, 1999.

[11] M. Ravikumar, R. Shukla, and A. A. Bothner-By, "Relaxation and dynamics of coupled spin systems subjected to continuous radio frequency fields," The Journal of Chemical Physics, vol. 95, no. 5, pp. 3092-3098, 1991.

[12] C. P. Slichter, Principles of Magnetic Resonance, SpringerVerlag, New York, NY, USA, 2nd edition, 1978. 